\section{Letter in reply to "Fertility preservation before an ABVD protocol: no new evidence to support changing the recommendations"}

\author{
Charlotte Sonigo $^{1,2}$, Nathalie Sermondade ${ }^{3}$ \& Michaël Grynberg ${ }^{* 1,2,4}$
}

First draft submitted: 22 November 2016; Accepted for publication: 29 November 2016; Accepted for publication: 8 December 2016

We carefully read Poirot et al.'s reply [1] to our article entitled "History of ABVD alters the number of oocytes vitrified after in vitro maturation in fertility preservation candidates" [2]. Overall, we agree with the comments. Indeed, our study is retrospective including two groups of patients having either received ABVD (adriamycin/ bleomycin/vinblastine/dacarbazine) chemotherapy for Hodgkin lymphoma $(\mathrm{n}=22)$ and controls suffering from breast cancer, without previous history of chemotherapy $(\mathrm{n}=44)$. All patients underwent transvaginal immature oocyte retrieval for urgent fertility preservation. Despite similar age, BMI, anti-Müllerian hormone level and antral follicle count, a lower number of cumulus oocyte complexes was recovered in patients with ovaries exposed to ABVD. However, oocyte maturation rates were comparable in both groups. These data lead us to question the relevance of oncofertility counseling and fertility preservation prior to ABVD treatment.

Regarding the comment on immature egg retrieval, and as mentioned by the authors, we have shown that both antral follicle count and serum anti-Müllerian hormone levels are tightly correlated with the number of cumulus oocyte complexes collected [3]. However, these data we obtained in in vitro maturation candidates without previous exposure to chemotherapy. It is conceivable that these correlations remain accurate even after history of chemotherapy but no strong data are currently available. In addition, we confirm that the practitioner experience represents a key element that might highly influence the output of the procedure. This point is easily explainable by the size of the small antral follicles punctured, which differs significantly from a conventional oocyte pick up following ovarian stimulation. In our study, all egg retrievals were performed by a single operator with great experience in in vitro maturation. Therefore, we hypothesized that postchemotherapy ovarian fibrosis, reported by Meirow et al. [4], might explain difficulties in oocyte collection. We are aware that these findings were not related to a specific type of drug and not systematically found, but it might constitute a viable reason for the lower output of egg retrieval in ABVD patients. However, due to the limited number of patients included in our study, a role of chance cannot be excluded. Moreover, as rightly emphasized by the authors, the cancer status might by itself impact the overall ovarian function $[5,6]$. From this point, we agree that Hodgkin lymphoma, in particular in advanced stages, might

'Department of Reproductive Medicine \& Fertility Preservation, Hôpital Jean-Verdier, Avenue du 14 Juillet, 93140 Bondy, France

2Université Paris XIII, 93000 Bobigny, France

${ }^{3}$ Department of Cytogenetic and Reproductive Biology, Hôpital Jean-Verdier, Avenue du 14 Juillet, 93140 Bondy, France ${ }^{4}$ Unité Inserm U1133, Université Paris-Diderot, 75013 Paris, France

*Author for correspondence: Tel.: +33 148026 856; Fax: +33 148026 860; michael.grynberg@aphp.fr

\section{KEYWORDS}

- fertility preservation • Hodgkin lymphoma • in vitro maturation 
affect more profoundly the ovarian follicular status than the breast cancer. However, we do not believe that when antral follicles are visible into the ovaries, the disease by itself, would reduce the chance to obtained the immature oocyte, implying a increased risk of empty follicle, a strongly debated concept otherwise. When our study was designed, the expected consequence of malignancies was rather a modification of the maturation rate due to the history of chemotherapy.

Regarding the effect of ABVD on the ovarian function, we fully agree with the authors. Data available in the literature confirm the low ovarian toxicity of this chemotherapy protocol [7-9]. Indeed, as reported in Harel's review, fertility following ABVD is probably not or poorly impacted. However, the authors do not recommend not referring patients for oncofertility counseling in particular when they are over the age of 30 years. Due to the risk, even low, of recurrence of the Hodgkin lymphoma or the possibility of developing secondary breast cancer, we still consider that fertility preservation should be discussed, which does not mean recommended. Indeed, women should be informed that in these clinical situations the possibility of offering them optimal fertility preservation might be compromised, either in case of therapeutic intensification following recent chemotherapy [10], and/or in emergency. Our data, with the underlined limitations, might constitute an additional argument for patient information.

Financial \& competing interests disclosure

The authors have no relevant affiliations or financial involvement with any organization or entity with a financial interest in or financial conflict with the subject matter or materials discussed in the manuscript. This includes employment, consultancies, honoraria, stock ownership or options, expert testimony, grants or patents received or pending, or royalties.

No writing assistance was utilized in the production of this manuscript.

\section{References}

1 Poirot C, Dhedin N, Brice P. Fertility preservation before an ABVD protocol: no new evidence to support changing the recommendations. Future Oncol. doi:10.2217/fon-2016-0441 (2016) (Epub ahead of print).

2 Sonigo C, Seroka A, Cedrin-Durnerin I, Sermondade N, Sifer C, Grynberg M. History of ABVD alters the number of oocytes vitrified after in vitro maturation in fertility preservation candidates. Future Oncol. 12(14), 1713-1719 (2016).

3 Sonigo C, Simon C, Boubaya M et al. What threshold values of antral follicle count and serum AMH levels should be considered for oocyte cryopreservation after in vitro maturation? Hum. Reprod. 31(7), 1493-1500 (2016).
4 Meirow D, Dor J, Kaufman B et al. Cortical fibrosis and blood-vessels damage in human ovaries exposed to chemotherapy. Potential mechanisms of ovarian injury. Hum. Reprod. 22(6), 1626-1633 (2007).

5 Lawrenz B, Fehm T, von Wolff M et al. Reduced pretreatment ovarian reserve in premenopausal female patients with Hodgkin lymphoma or non-Hodgkin-lymphoma evaluation by using antimüllerian hormone and retrieved oocytes. Fertil. Steril. 98(1), 141-144 (2012).

6 Lekovich J, Lobel ALS, Stewart JD, Pereira N, Kligman I, Rosenwaks Z. Female patients with lymphoma demonstrate diminished ovarian reserve even before initiation of chemotherapy when compared with healthy controls and patients with other malignancies. J. Assist. Reprod. Gent. 33, 657-662 (2016).
7 Decanter C, Morschhauser F, Pigny P, Lefebvre C, Gallo C, Dewailly D. AntiMüllerian hormone follow up in young women treated by chemotherapy for lymphoma: preliminary results. Reprod. Biomed. Online 20 (2), 280-285 (2010).

8 Harel S, Fermé C, Poirot C. Management of fertility in patients treated for Hodgkin's lymphoma. Haematologica 96(11), 1692-1699 (2011).

9 Rosendahl M, Andersen CY, Ernst E et al. Ovarian function after removal of an entire ovary for cryopreservation of pieces of cortex prior to gonadotoxic treatment: a follow-up study. Hum. Reprod. 23(11), 2475-2483 (2008).

10 Amon J, Meirow D, Lewis-Roness H, Omoy A. Genetic and teratogenic effects of cancer treatments on gametes and embryos. Hum. Reprod. Update 7(4), 394-403 (2001). 